\title{
Verrucous Eccrine Angiomatous Hamartoma
}

\author{
ali sadeghinia ${ }^{1}$, elham mazaherpour ${ }^{2}$, and Alireza Ghanadan ${ }^{1}$ \\ ${ }^{1}$ Tehran University of Medical Sciences \\ ${ }^{2}$ Affiliation not available
}

April 28, 2020

\begin{abstract}
Eccrine Angiomatous Hamartoma (EAH) is a rare malformation with the proliferation of eccrine glands and other dermal structures. It typically presents as a solitary lesion on extremities. We report a case of Verrucous EAH on the heel of a 27-years old man which is an uncommon presentation of this tumor
\end{abstract}




\section{Verrucous Eccrine Angiomatous Hamartoma}

Ali Sadeghinia ${ }^{1}$; Elham Mazaherpour ${ }^{2}$; Alireza Ghanadan ${ }^{3}$

${ }^{1}$ Assistant professor of dermatology at university hospital of Razi, Tehran medical university, Tehran, Iran address: Vahdat islami square, Razi hospital, Tehran, Iran Postal address: 1199663911/ phone number: 98-9128142874 (sadeghinia2000@yahoo.com)

${ }^{2}$ M.D, Residency in dermatology at university hospital of Razi, Tehran medical university, Tehran, Iran (elham.md900@gmail.com)

${ }^{3}$ Assistant professor of dermatopathology at university hospital of Razi, Tehran medical university, Tehran, Iran (dermpath101@gmail.com) Vahdat islami square,Razi hospital, Tehran, Iran Postal address: 1199663911/ 
phonenumber: 98-9153178001

Corresponding Author:

Elham Mazaherpour, MD

Dermatology Hospital of Razi

Vahdat islami square,Razi hospital, Tehran, Iran

Tel: +98-9124681594

Fax: +98-2155620300

Postal address: 1199663911

Email: elham.md900@gmail.com

Abstract

Eccrine Angiomatous Hamartoma (EAH) is a rare malformation with the proliferation of eccrine glands and other dermal structures. It typically presents as a solitary lesion on extremities which appears at birth or during childhood. It is generally asymptomatic but may be associated with hyperhidrosis or pain. We report a case of Verrucous EAH on the heel of a 27-years old man which is an uncommon presentation of this tumor and review the clinical characteristics, histologic findings and prognosis of this rare condition.

Key words: eccrine; angiomatous; hamartoma; verrucous

Key clinical message: Eccrine Angiomatous Hamartoma (EAH) is a rare condition. It can be verrucous which is an uncommon feature of the tumor. Our case is unique because of its verrucous feature

Introduction

Eccrine angiomatous hamartoma is a rare hamartoma that comprises the proliferation of the eccrine glands within a vascular stroma and proliferation of other dermal structures such as hair, adipose tissue $(1,2)$. epidermal hyperplasia has also been noted. (3) The tumor often occurs at birth or childhood as a solitary plaque or nodule on the extremities. (2) The tumor manifestations range from a simple angiomatous nodules to erythematous plaques. (1) Generally, the lesion is asymptomatic but pain, hyperhidrosis, and hypertrichosis occurs in some cases. $(4,5)$ In this article, we report a case of Verrucous EAH in the heel of a 27-years old man.

Case report

A 27-year old previously healthy man presented with a lesion on her right heel from 2 years of age. His lesion had been grown with the time. It was not painful. The only symptom the patient complained was sweating of the lesion when he was hot. He was otherwise developmentally normal and had no musculoskeletal abnormalities. He denied the use of medication and no one else among her family had a similar lesion. In the physical examination a solitary bruise-like verrucous $4^{*} 6 \mathrm{~cm}$ palpable plaque was seen in the right heel (figure 1). The lesion was well-circumscribed and there was no pain upon compression.

A skin biopsy was done. Histopathologic examination showed a lobular compartment with increased eccrine glands and vascular channels juxtaposed to the nerve bundles in deep dermis (figure 2a-2b). The vascular component was composed of small capillaries with variable dilation associated with mature-appearing eccrine glands in a lobular arrangement. Overlying epidermis revealed hyperkeratosis, moderate acanthosis and low papillomatosis with irregular rete ridges (figure 2c). The patient declined surgical treatment.

\section{Discussion}

First Lotzbeck reported EAH in 1859 with an angiomatous lesion on the cheek of a child. (1) The tumor presents equally in males and females and approximately a third of cases occur from birth to childhood. Few studies have reported the lesions that appear in adulthood. (1, 2) It typically manifests as a single, 
flesh-colored, blue-brown or reddish papule, plaque or nodule, although uncommonly multiple lesions or hyperkeratotic verrucous variants are also seen. (4) The tumor usually locates on acral sites, especially on palms and soles. (6) The face, neck, abdomen, scrotal region and back can also be involved. (4)

hyperhidrosis and pain are common symptoms that can be spontaneous or after pressure. Probably pain appears due to nerve fiber involvement and local temperature elevation in the lesion stimulates eccrine glands which leads to hyperhidrosis. (1)

Most cases enlarge with body size and sudden enlargement can occur during pregnancy and puberty probably due to hormonal influence. $(4,7)$

biopsy is needed for a definite diagnosis of EAH. histopathologic criteria are eccrine glands and vascular elements proliferation and also other dermal structures such as apocrine glands, pilar, mucinous, lipomatous, and lymphatic. (6) The tumor is often well defined in the middle or deep dermis with unremarkable epidermis. $(4,6)$ cases of EAH with overlying verrucous epiderm have been reported in rare cases. $(3,6)$ We report another rare case in this article with an interesting presentation. In verrucous EAH epiderm shows hyperkeratosis, acanthosis, and papillomatosis. (3)

Immunohistochemical stains (IHC) with Pancytokeratin and CD31 may show eccrine glands and capillaries positivity, respectively. (2) The main differential diagnosis are other vascular malformations, smooth muscle hamartoma, glomus tumor and tufted angioma $(1,6)$.treatment options are: surgery for small lesion (1), laser depilation for hypertrichosis and botulinum toxin for hyperhidrosis. (6)

1.Pinbeiro A, Rodrigues H. Eccrine Angiomatous hamartoma: case report and clinical, pathologic and ultrasonographic studies. An Bras Dermatol. 2005;80(4).

2.Fathaddin AA, Alhumidi AA. Eccrine angiomatous hamartoma, with verrocous hemangioma-like features: A case report. Int J Health Sci (Qassim). 2013;7(1):103-6.

3.Galan A, McNiff JM. Eccrine angiomatous hamartoma with features resembling verrucous hemangioma. J Cutan Pathol. 2007;34 Suppl 1:68-70.

4.Lin YT, Chen CM, Yang CH, Chuang YH. Eccrine angiomatous hamartoma: a retrospective study of 15 cases. Chang Gung Med J. 2012;35(2):167-77.

Dadlani C, Orlow SJ. Eccrine angiomatous hamartoma. Dermatol Online J. 2006;12(5):9.5

6.Tantanasrigul P, Kootiratrakarn T, Wessagowit V, Kattipathanapong P, Sudtikoonaseth P. Eccrine Angiomatous Hamartoma in an Adolescent. Case Rep Dermatol. 2015;7(3):233-6

7.Apte A, Nema P, Bandi A. Eccrine angiokeratomatous hamartoma: case report of a 1.5-year girl. J Surg Case Rep. 2017;2017(7):rjx133. 

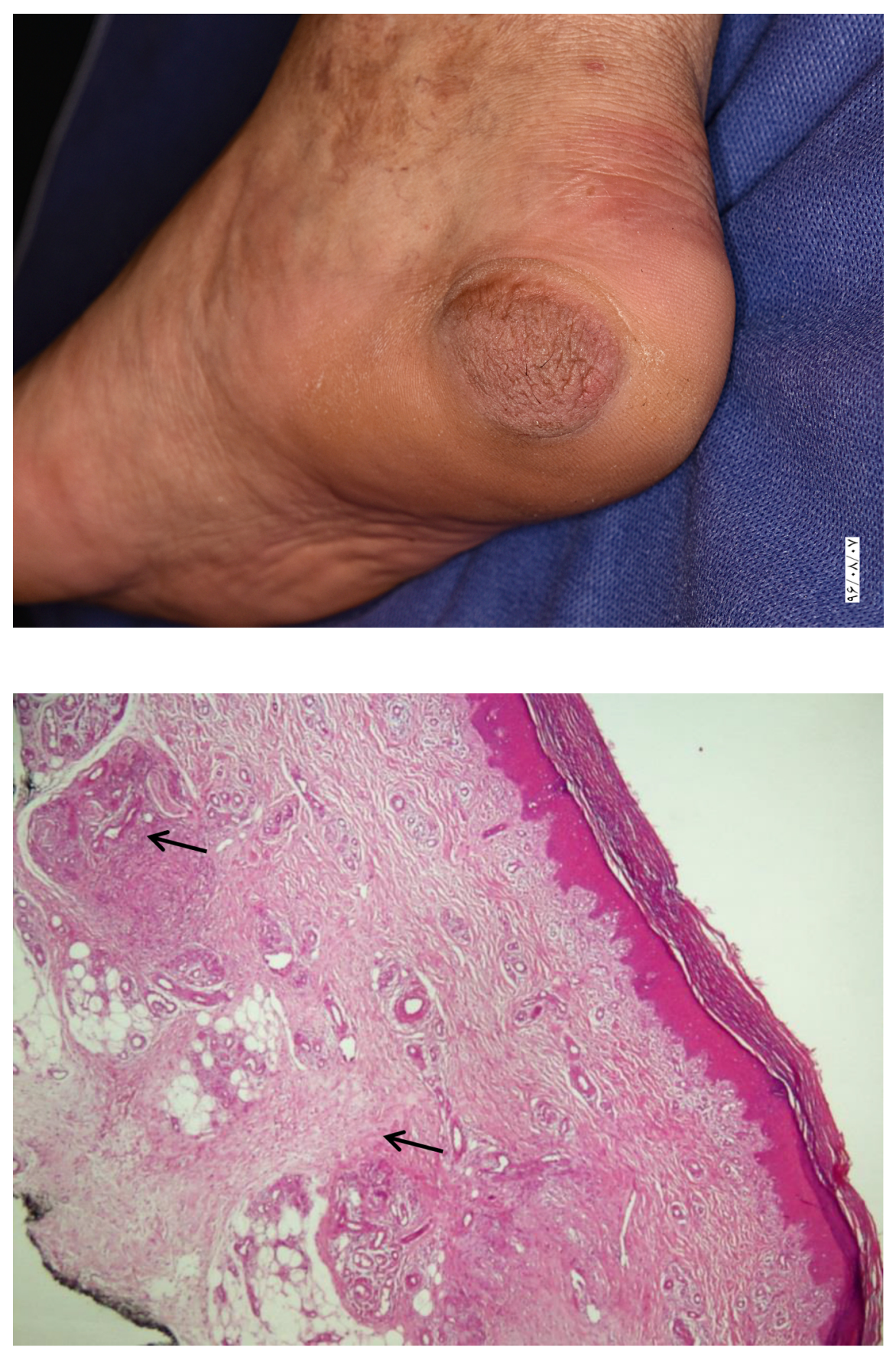

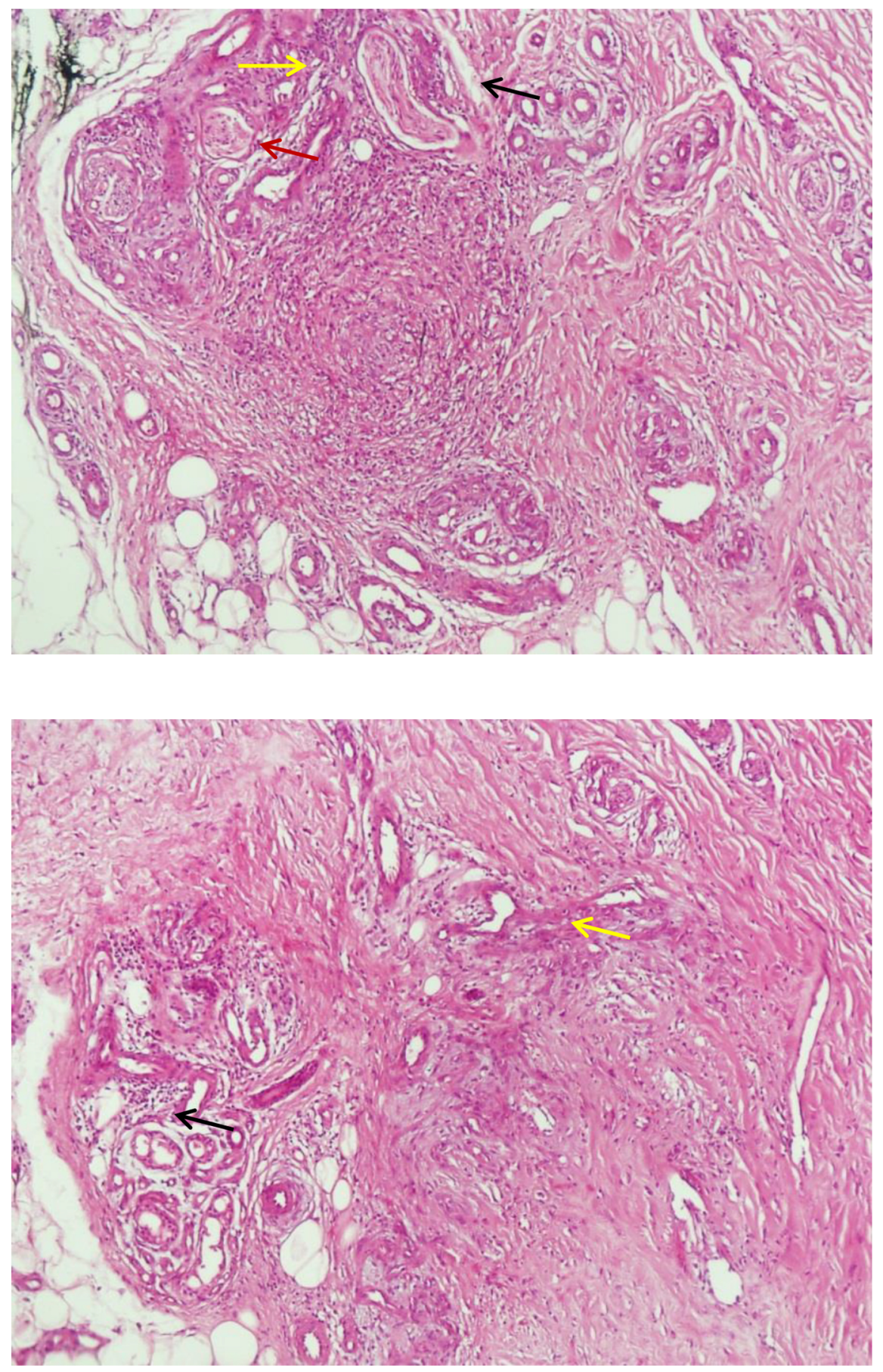